ANNALES

POLONICI MATHEMATICI

$94.2(2008)$

\title{
Dynamics and density evolution in piecewise deterministic growth processes
}

\author{
by MiCHAEL C. MACKEY (Montreal) \\ and MARTA Tyran-Kamińska (Katowice)
}

Dedicated to the memory of Andrzej Lasota (1932-2006)

\begin{abstract}
A new sufficient condition is proved for the existence of stochastic semigroups generated by the sum of two unbounded operators. It is applied to one-dimensional piecewise deterministic Markov processes, where we also discuss the existence of a unique stationary density and give sufficient conditions for asymptotic stability.
\end{abstract}

1. Introduction. The development of cell cycle models to account for the statistical properties of division dynamics in populations of cells inevitably led to the consideration of stochastically perturbed dynamical systems $[5,11,13,23,24]$. These applied considerations have been followed by work on the behaviour of Poisson driven dynamical systems in a pure mathematical context $[14,21]$. More recently other areas of application related to the role of intrinsic (as opposed to extrinsic) noise in gene regulatory dynamics $[9,15,4]$ have made the understanding of stochastic perturbations of dynamical systems of more than passing interest.

We were originally motivated by the work of Lasota et al. [13] who considered a (biological) system which produces "events" and has an internal or physiological time in addition to the laboratory time $t$. We denote this internal time by $\tau$ to distinguish from the time $t$. When an event appears the physiological time $\tau=\tau_{e}$ is reset to $\tau=0$. We assume that the rate $d \tau / d t$ depends on the amount of an "activator" which we denote by $a$. Thus we have

$$
\frac{d \tau}{d t}=\varphi(a), \quad \varphi \geq 0
$$

2000 Mathematics Subject Classification: Primary 47D06; Secondary 60J25.

Key words and phrases: first order partial differential equation, stochastic semigroup, asymptotic stability, invariant density.

M. Tyran-Kamińska is the corresponding author. 
The activator is produced by a dynamics described by the differential equation

$$
\frac{d a}{d t}=g(a),
$$

where $g \geq 0$ is a continuous function on an open interval that may or may not be bounded. When an event is produced at a time $\tau_{e}$ and activator level $a_{e}$, then a portion $\varrho\left(a_{e}\right)$ of $a_{e}$ is consumed so the level of the activator after the event is then

$$
\sigma\left(a_{e}\right):=a_{e}-\varrho\left(a_{e}\right) .
$$

We also allow the possibility that the portion $\varrho\left(a_{e}\right)$ depends on an environmental or external factor so that $\varrho$ is a function of two variables $\varrho\left(a_{e}, \theta_{e}\right)$, where $\theta_{e} \in \Theta$ is distributed according to some probability measure $\nu$ on $\Theta$. The solution of $(1.2)$ with the initial condition $a(0)=x$ will be denoted by

$$
a(t)=\pi_{t} x
$$

and we assume that it is defined for all $t \geq 0$. Then the solution of equation (1.1) with the initial condition $\tau(0)=0$ is given by

$$
\tau(t)=\int_{0}^{t} \varphi\left(\pi_{r} x\right) d r
$$

It is reasonable to require that also $\tau(t)$ is finite for all $t \geq 0$.

Lasota et al. [13] studied the statistical behaviour of a sequence of such events occurring at random times

$$
0=t_{0}<t_{1}<\cdots<t_{n}<\cdots
$$

and denoted $a_{n}=a\left(t_{n}\right)$ to find

$$
a_{n+1}=T\left(a_{n}, \tau_{n}\right), \quad \text { where } \quad \tau_{n}=\int_{t_{n-1}}^{t_{n}} \varphi\left(\pi_{s-t_{n-1}} a_{n-1}\right) d s
$$

were exponentially distributed independent random variables, giving a relation between successive activator levels at event occurrence and studying a discrete time system with stochastic perturbations by the $\tau_{n}$. Here we have extended these considerations to a continuous time situation by examining what happens at all times $t$ and not merely at $t_{0}, t_{1}, t_{2}, \ldots$ Thus we arrive at a continuous time piecewise deterministic Markov process, whose sample paths between the jump times $t_{0}, t_{1}, \ldots$ are given by the solution of $(1.2)$ and at the jump times the state of the process is selected according to a jump stochastic kernel, which is the transition probability function for (1.3). This leads us to study evolution equations of the form 


$$
\frac{\partial u}{\partial t}=A_{0} u-\varphi u+P(\varphi u), \quad \text { where } \quad A_{0} u(x)=-\frac{d}{d x}(g(x) u(x)),
$$

on the space of integrable functions $L^{1}$, where $P$ is a stochastic operator on $L^{1}$ corresponding to the jump stochastic kernel and $\varphi$ need not be bounded. We supplement (1.5) with the initial condition $u(0)=u_{0}$ which is the density of the distribution of the initial amount of the activator.

Let us write

$$
A u=A_{0} u-\varphi u \quad \text { and } \quad \mathcal{C} u=A u+P(\varphi u) .
$$

If $\varphi$ is unbounded then $\mathcal{C}$ is the sum of two unbounded operators, so the existence and uniqueness of solutions to the Cauchy problem in $L^{1}$ is problematic; (1.5) may have multiple solutions [2]. We make use of perturbation results for positive semigroups on $L^{1}$-spaces which go back to [10] (see Section 3), from which it follows that the operator $\mathcal{C}$ has an extension $C$ generating a positive contraction semigroup $\{P(t)\}_{t \geq 0}$ provided that the operator $A$ is the infinitesimal generator of a positive contraction semigroup on $L^{1}$ and $\mathcal{C}$ is defined on the domain of $A$. In general, if the closure of $\mathcal{C}$ is the generator $C$ of $\{P(t)\}_{t \geq 0}$ then the Cauchy problem is uniquely solved and $\{P(t)\}_{t \geq 0}$ is a stochastic semigroup. In Section 3 we prove a new sufficient condition for uniqueness and in Section 5 we show that if the discrete process has a strictly positive stationary density then uniqueness holds. This simplifies the analysis of (1.5) when compared with the approach in [2], and allows us to investigate both the uniqueness of solutions and their asymptotic properties.

The outline of this paper is as follows. We recall basic definitions and fundamental theorems from the theory of stochastic operators and semigroups in Section 2 and perturbation results for positive semigroups on $L^{1}$-spaces in Section 3, which closes with the proof of our main general result (Theorem 3). In Section 4 we prove that the operators $A_{0}$ and $A$ defined on suitable domains are generators. In Section 5 we show the applicability of Theorem 3 to equation (1.5) when $P$ is an arbitrary stochastic operator and also give sufficient conditions for asymptotic stability. In Section 6 we let the operator $P$ have a definite form that fits directly into our framework, and give several concrete examples drawn from work on the regulation of the cell cycle as well as classical integro-differential equations. In Section 7 we extend our results to the situation in which there is degradation (as opposed to growth) and illustrate their applicability using models for the stochastic regulation of gene expression.

In a companion paper [22], these and other results are placed in the general context of semigroup theory and probability theory with applications to piecewise deterministic Markov process without "active boundaries". There we also prove that if the semigroup $\{P(t)\}_{t \geq 0}$ is stochastic then (1.5) is the corresponding evolution equation for densities of such processes. 
2. Stochastic operators and semigroups. Let $(E, \mathcal{E}, m)$ be a $\sigma$-finite measure space. We denote by $D$ the set of all densities on $E$, i.e.

$$
D=\left\{u \in L^{1}: u \geq 0,\|u\|=1\right\},
$$

where $\|\cdot\|$ is the norm in $L^{1}=L^{1}(E, \mathcal{E}, m)$. A linear operator $P: L^{1} \rightarrow L^{1}$ such that $P(D) \subset D$ is called stochastic or Markov [12].

Let $P: L^{1} \rightarrow L^{1}$ be a stochastic operator. A density $u$ is said to be invariant or stationary for $P$ if $P u=u$. We say that $P$ overlaps supports if for every $u, v \in D$ there is a positive integer $n \geq 1$ such that

$$
m\left(\operatorname{supp} P^{n} u \cap \operatorname{supp} P^{n} v\right)>0,
$$

where the support of $u \in L^{1}$ is defined up to a set of measure zero by the formula supp $u=\{x \in E: u(x) \neq 0\}$. Note that if $P$ overlaps supports then it can have at most one invariant density [18, proof of Corollary 1].

Let $\mathcal{J}: E \times \mathcal{E} \rightarrow[0,1]$ be a stochastic transition kernel, i.e. $\mathcal{J}(x, \cdot)$ is a probability measure for each $x \in E$ and the function $x \mapsto \mathcal{J}(x, B)$ is measurable for each $B \in \mathcal{E}$, and let $P$ be a stochastic operator on $L^{1}$. If

$$
\int_{E} \mathcal{J}(x, B) u(x) m(d x)=\int_{B} P u(x) m(d x) \quad \text { for all } B \in \mathcal{E}, u \in D(m),
$$

then $P$ is called the transition operator corresponding to $\mathcal{J}$.

If $\mathcal{J}(x, B)=1_{T^{-1}(B)}(x)$ for $x \in E, B \in \mathcal{E}$, where $T: E \rightarrow E$ is a nonsingular measurable transformation, i.e. $m\left(T^{-1}(B)\right)=0$ for all $B \in \mathcal{E}$ such that $m(B)=0$, then there exists a unique stochastic operator $P$ on $L^{1}$ satisfying (2.1), and it is called the Frobenius-Perron operator corresponding to $T$.

A stochastic operator $P$ on $L^{1}$ is called partially integral or partially kernel if there exists a measurable function $p: E \times E \rightarrow[0, \infty)$ such that

$$
\int_{E} \int_{E} p(x, y) m(d y) m(d x)>0 \quad \text { and } \quad P u(x) \geq \int_{E} p(x, y) u(y) m(d y)
$$

for every density $u$. If, additionally,

$$
\int_{E} p(x, y) m(d x)=1, \quad y \in E,
$$

then $P$ corresponds to the stochastic kernel

$$
\mathcal{J}(x, B)=\int_{B} p(y, x) m(d y), \quad x \in E, B \in \mathcal{E},
$$

and we simply say that $P$ has kernel $p$.

A strongly continuous semigroup $\{P(t)\}_{t \geq 0}$ on $L^{1}$ is called a stochastic semigroup or Markov semigroup if $P(t)$ is a stochastic operator for all $t \geq 0$. A density $u_{*}$ is called invariant or stationary for $\{P(t)\}_{t \geq 0}$ if $P(t) u_{*}=u_{*}$ for every $t \geq 0$. 
A stochastic semigroup $\{P(t)\}_{t \geq 0}$ is called asymptotically stable if there is a stationary density $u_{*}$ such that

$$
\lim _{t \rightarrow \infty}\left\|P(t) u-u_{*}\right\|=0 \quad \text { for } u \in D,
$$

and it is called partially integral if, for some $t_{0}>0$, the operator $P\left(t_{0}\right)$ is partially integral.

TheOREM 1 ([17]). Let $\{P(t)\}_{t \geq 0}$ be a partially integral stochastic semigroup. Assume that the semigroup has only one invariant density $u_{*}$. If $u_{*}>0$ a.e. then the semigroup is asymptotically stable.

3. Perturbation results in $L^{1}$. Let $\left(A_{0}, \mathcal{D}\left(A_{0}\right)\right)$ be the infinitesimal generator of a stochastic semigroup, $\varphi \geq 0$ be a measurable function, and

$$
L_{\varphi}^{1}=\left\{u \in L^{1}: \int_{E} \varphi(x)|u(x)| m(d x)<\infty\right\} .
$$

Let $P$ be a stochastic operator on $L^{1}$ and let the operators $A$ and $\mathcal{C}$, as given in (1.6), be defined on $\mathcal{D}(A) \subseteq \mathcal{D}\left(A_{0}\right) \cap L_{\varphi}^{1}$. Assume that $(A, \mathcal{D}(A))$ is the infinitesimal generator of a positive strongly continuous contraction semigroup $\{S(t)\}_{t \geq 0}$ on $L^{1}$. Then it is known $[10,25,1,2]$ that there exists a positive strongly continuous contraction semigroup $\{P(t)\}_{t \geq 0}$ on $L^{1}$ satisfying the following:

(1) the infinitesimal generator $C$ of $\{P(t)\}_{t \geq 0}$ is an extension of $\mathcal{C}$, i.e. $\mathcal{D}(A) \subseteq \mathcal{D}(C)$ and $C u=\mathcal{C} u$ for $u \in \mathcal{D}(A)$;

(2) if $\{\bar{P}(t)\}_{t \geq 0}$ is another semigroup generated by an extension of $\mathcal{C}$ then $\bar{P}(t) u \geq P(t) u$ for all $u \in L^{1}, u \geq 0$, i.e. $\{P(t)\}_{t \geq 0}$ is the minimal semigroup;

(3) the generator $C$ is characterized by

$$
R(\lambda, C) u=\lim _{N \rightarrow \infty} R(\lambda, A) \sum_{n=0}^{N}(P(\varphi R(\lambda, A)))^{n} u, \quad u \in L^{1}, \lambda>0,
$$

where $R(\lambda, \cdot)$ is the resolvent operator;

(4) the semigroup $\{P(t)\}_{t \geq 0}$ satisfies the equation

$$
P(t) u=S(t) u+\int_{0}^{t} P(t-s) P(\varphi S(s) u) d s, \quad u \in \mathcal{D}(A) .
$$

We cannot conclude, in general, that the semigroup $\{P(t)\}_{t \geq 0}$ is stochastic [10, Example 4.3]. Discussing various conditions for this to hold has been a major objective of study $[10,25,1,7,2]$ and leads to the following result.

THEOREM 2. If for some $\lambda>0$,

$$
\lim _{n \rightarrow \infty}\left\|(P(\varphi R(\lambda, A)))^{n} u\right\|=0 \quad \text { for all } u \in L^{1}
$$


then $\{P(t)\}_{t \geq 0}$ is a stochastic semigroup and its generator $C$ is the closure of the operator $(\mathcal{C}, \mathcal{D}(A))$.

We now prove our main result.

THeOREM 3. If for some $\lambda>0$ there is $v \in L^{1}$ such that $v>0$ a.e. and $P(\varphi R(\lambda, A)) v \leq v$, then condition (3.3) holds.

Proof. We can assume that $v$ is a density. Set $K_{\lambda}=P(\varphi R(\lambda, A))$. The operator $K_{\lambda}$ is a positive contraction. Since $K_{\lambda} v \leq v$, the sequence $K_{\lambda}^{n} v$ is strongly convergent in $L^{1}$ to a fixed point $u_{*}$ of the operator $K_{\lambda}$. From [2, Theorem 4.3] it follows that $u_{*}=0$. For any density $u$ we have

$$
0 \leq K_{\lambda}^{n} u_{k} \leq k K_{\lambda}^{n} v, \quad \text { where } \quad u_{k}=\min \{u, k v\}, k, n \geq 1 .
$$

Since $\left\|u_{k}-u\right\| \rightarrow 0$ as $k \rightarrow \infty$, this completes the proof of (3.3).

4. The semigroup $\{S(t)\}_{t \geq 0}$. Let $E$ be an open interval in $\mathbb{R}$, bounded or unbounded, $\mathcal{E}=\mathcal{B}(E)$ and $m$ be the Lebesgue measure. We shall denote by $L_{\text {loc }}^{1}$ the space of all Borel measurable functions on $E$ which are integrable on compact subsets of $E$ and by $A C$ the space of all absolutely continuous functions on $E$. We assume from now on that $E=\left(d_{0}, d_{1}\right)$, where $-\infty \leq$ $d_{0}<d_{1} \leq \infty, g: E \rightarrow \mathbb{R}$ is a continuous strictly positive function, and $\varphi \in L_{\text {loc }}^{1}$ is nonnegative. In this section we study the first order differential operators $A_{0}$ and $A$ which are meaningful for any function $u \in L_{\text {loc }}^{1}$ for which $g u \in A C$. We will define the operators $A_{0}$ and $A$ on suitable domains $\mathcal{D}\left(A_{0}\right)$ and $\mathcal{D}(A)$ so that they are generators of corresponding semigroups as described in Section 3.

Since $1 / g, \varphi / g \in L_{\mathrm{loc}}^{1}$, we can define

$$
G(x)=\int_{x_{0}}^{x} \frac{1}{g(z)} d z \quad \text { and } \quad Q(x)=\int_{x_{1}}^{x} \frac{\varphi(z)}{g(z)} d z,
$$

where $x_{0}=d_{0}$ and $x_{1}=d_{0}$ when the integrals exist for all $x$; otherwise, $x_{0}, x_{1}$ are any points in $E$. The function $G$ is strictly monotone, continuously differentiable on $E, G\left(d_{0}\right) \in\{0,-\infty\}$, and $G\left(d_{1}\right)$ is either finite or equal to $+\infty$. The function $Q$ is monotone with $Q\left(d_{0}\right) \in\{0,-\infty\}$ and $Q\left(d_{1}\right)$ is either finite or equal to $+\infty$. $G$ is invertible with $G^{-1}$ well defined on $G(E)$. If $G(E) \neq \mathbb{R}$, then we extend $G^{-1}$ continuously so that $G^{-1}(\mathbb{R} \backslash G(E))=$ $\left\{d_{0}, d_{1}\right\}$. The formula

$$
a(t, x)=G^{-1}(G(x)+t), \quad x \in E, t \in \mathbb{R},
$$

defines a monotone continuous function in each variable with values in $[-\infty, \infty]$ which is a solution of (1.2). If $G\left(d_{1}\right)=+\infty$ then $\pi_{t} x=a(t, x)$ is finite for all $t \geq 0, x \in E$, and $\pi_{t}(E) \subseteq E, t \geq 0$. In the case when 
$\left|G\left(d_{0}\right)\right|=G\left(d_{1}\right)=\infty$ the value $a(t, x)$ is finite for all $t \in \mathbb{R}$ and $x \in E$, so that we have, in fact, a flow $\pi_{t}$ on $E$ such that $\pi_{t}(E)=E$.

For $t>0$ we define the operators $P_{0}(t)$ and $S(t)$ on $L^{1}$ by

$$
\begin{aligned}
& P_{0}(t) u(x)=\mathbf{1}_{E}\left(\pi_{-t} x\right) u\left(\pi_{-t} x\right) \frac{g\left(\pi_{-t} x\right)}{g(x)}, \quad x \in E, u \in L^{1}, \\
& S(t) u(x)=e^{Q\left(\pi_{-t} x\right)-Q(x)} P_{0}(t) u(x), \quad x \in E, u \in L^{1} .
\end{aligned}
$$

Theorem 4. If $G\left(d_{1}\right)=+\infty$ then $\left\{P_{0}(t)\right\}_{t \geq 0}$ is a stochastic semigroup and $\{S(t)\}_{t \geq 0}$ is a positive strongly continuous contraction semigroup on $L^{1}$.

Proof. $P_{0}(t)$ is a stochastic operator because it is the Frobenius-Perron operator for the transformation $x \mapsto \pi_{t} x$. Since $Q$ is nondecreasing and $t \mapsto \pi_{t} x$ is increasing, we always have

$$
e^{Q\left(\pi_{-t} x\right)-Q(x)} \mathbf{1}_{E}\left(\pi_{-t} x\right) \leq \mathbf{1}_{E}\left(\pi_{-t} x\right) .
$$

Hence $S(t)$ is a positive contraction. To check the semigroup property observe that if $x \in E$ and $\pi_{-s-t} x \in E$, then $\pi_{s}\left(\pi_{-s-t} x\right) \in E$, by assumption, and thus $\pi_{s}\left(\pi_{-s-t} x\right)=\pi_{-t} x \in E$. Furthermore, if $x \in E$ and $\pi_{-t} x \in E$ then $\pi_{-s}\left(\pi_{-t} x\right)=\pi_{-s-t} x$. Hence

$$
\mathbf{1}_{E}\left(\pi_{-t} x\right) \mathbf{1}_{E}\left(\pi_{-s}\left(\pi_{-t} x\right)\right)=\mathbf{1}_{E}\left(\pi_{-s-t} x\right),
$$

which shows that $S(t) S(s) u(x)=S(s+t) u(x)$ for $t, s \geq 0, x \in E, u \in L^{1}$. Finally, we must show that $\{S(t)\}_{t \geq 0}$ is strongly continuous. Let $u \in C_{c}(E)$, where $C_{c}(E)$ is the space of continuous functions which are equal to zero near boundaries. For every $x \in E$ and all sufficiently small $t>0$ we have $\mathbf{1}_{E}\left(\pi_{-t} x\right)=1$ and $\pi_{-t} x \rightarrow x$ as $t \downarrow 0$. Consequently,

$$
\lim _{t \downarrow 0} S(t) u(x)=u(x), \quad x \in E,
$$

which, by the Lebesgue dominated convergence theorem, implies

$$
\lim _{t \downarrow 0}\|S(t) u-u\|=0 .
$$

Since $C_{c}(E)$ is a dense subset of $L^{1}$, this shows the strong continuity of the semigroup $\{S(t)\}_{t \geq 0}$. If we take $\varphi \equiv 0$ then $S(t)=P_{0}(t)$ and we get the claim for $\left\{P_{0}(t)\right\}_{t \geq 0}$.

Now we identify the generators of the semigroups from Theorem 4 . The maximal domain of $A_{0}$ in $L^{1}$ consists of all functions $u \in L^{1}$ such that $A_{0} u \in L^{1}$. Then the integrability of $A_{0} u$ implies existence of the finite limits

$$
l_{0}(u):=\lim _{x \downarrow d_{0}} g(x) u(x) \quad \text { and } \quad l_{1}(u):=\lim _{x \uparrow d_{1}} g(x) u(x) .
$$

A necessary condition for $A_{0}$ to generate a stochastic semigroup is that the limits $l_{i}$ are equal. Recall that $u \in L_{\varphi}^{1}$ if and only if $u \in L^{1}$ and $\varphi u \in L^{1}$. 
Theorem 5. If $G\left(d_{1}\right)=+\infty$ then the operator $A_{0}$ defined on the domain

$$
\mathcal{D}\left(A_{0}\right)=\left\{u \in L^{1}: g u \in A C, A_{0} u \in L^{1}, \lim _{x \downarrow d_{0}} g(x) u(x)=0\right\}
$$

is the generator of the semigroup $\left\{P_{0}(t)\right\}_{t \geq 0}$, and the operator $A$ defined on $\mathcal{D}(A)=\mathcal{D}\left(A_{0}\right) \cap L_{\varphi}^{1}$ is the generator of $\{\bar{S}(t)\}_{t \geq 0}$.

Before we give the proof of Theorem 5 we first provide general properties of the operator $A$. Let $\lambda>0$. Define the function $r_{\lambda}: E \times E \rightarrow[0, \infty)$ by

$$
r_{\lambda}(x, y)=\mathbf{1}_{\left(d_{0}, x\right)}(y) \frac{e^{Q_{\lambda}(y)-Q_{\lambda}(x)}}{g(x)}, \quad \text { where } \quad Q_{\lambda}(z)=\lambda G(z)+Q(z),
$$

and the positive linear operator $R_{\lambda}: L^{1} \rightarrow L^{1}$ by

$$
R_{\lambda} v(x)=\int_{d_{0}}^{d_{1}} r_{\lambda}(x, y) v(y) d y .
$$

Lemma 1. Let $\lambda>0$. The operator $R_{\lambda}$ satisfies

$$
\lambda\left\|R_{\lambda} v\right\| \leq\|v\|, \quad v \in L^{1} .
$$

For every $v \in L^{1}$ we have $g R_{\lambda} v \in A C$ and the function $u=R_{\lambda} v$ is a particular solution in $L^{1}$ of the equation

$$
\lambda u-A u=v .
$$

Proof. $R_{\lambda}$ is an integral operator with nonnegative measurable kernel $r_{\lambda}$. Observe that

$$
\lambda r_{\lambda}(x, y) \leq \mathbf{1}_{\left(d_{0}, x\right)}(y) \frac{\lambda}{g(x)} e^{\lambda(G(y)-G(x))} \quad \text { for } x, y \in E .
$$

Thus for every $y \in E$ we have

$$
\lambda \int_{d_{0}}^{d_{1}} r_{\lambda}(x, y) d x \leq 1-e^{-\lambda\left(G\left(d_{1}\right)-G(y)\right)} \leq 1,
$$

because $G\left(d_{1}\right) \geq G(y)$, showing $\lambda\left\|R_{\lambda}\right\| \leq 1$. Since $e^{-Q} \in A C$, we can write

$$
A u(x)=-e^{-Q(x)} \frac{d}{d x}\left(g(x) u(x) e^{Q(x)}\right)
$$

for all $u$ such that $g u \in A C$. We have

$$
g(x) R_{\lambda} v(x)=e^{-Q_{\lambda}(x)} \int_{d_{0}}^{x} e^{Q_{\lambda}(y)} v(y) d y
$$

and $Q_{\lambda}(y) \leq Q_{\lambda}(\alpha)$ for every $y \leq \alpha<d_{1}$, thus the function $e^{Q_{\lambda}} v$ is integrable on intervals $\left(d_{0}, \alpha\right]$ for every $\alpha<d_{1}$. Hence, $g R_{\lambda} v \in A C$ and $R_{\lambda} v$ satisfies (4.8). 
Lemma 2. If $Q_{\lambda}\left(d_{1}\right)=+\infty$ then $R_{\lambda}\left(L^{1}\right) \subseteq \mathcal{D}(A)$ and

$$
R_{\lambda}(\lambda u-A u)=u \quad \text { for } u \in \mathcal{D}(A) .
$$

Proof. First we show that $R_{\lambda}\left(L^{1}\right) \subseteq \mathcal{D}(A)$. Let $v \in L^{1}$. Then $g R_{\lambda} v \in$ $A C$,

$$
\left|g(x) R_{\lambda} v(x)\right| \leq \int_{d_{0}}^{d_{1}}\left|g(x) r_{\lambda}(x, y)\right||v(y)| d y \quad \text { for } x \in E,
$$

and $\left|g(x) r_{\lambda}(x, y)\right| \leq \mathbf{1}_{\left(d_{0}, x\right)}(y)$ for all $x, y \in E$. From the definition of $r_{\lambda}$ and the assumption $Q_{\lambda}\left(d_{1}\right)=+\infty$ it follows that

$$
\lim _{x \rightarrow d_{i}}\left|g(x) r_{\lambda}(x, y)\right|=0 \quad \text { for } y \in E .
$$

By the Lebesgue dominated convergence theorem, $l_{i}\left(R_{\lambda} v\right)=0, i=0,1$, for all $v \in L^{1}$. Since $|v| \in L^{1}$, the function $R_{\lambda}|v|$ is a particular solution of

$$
(\lambda+\varphi) R_{\lambda}|v|=|v|+A_{0}\left(R_{\lambda}|v|\right)
$$

and $l_{i}\left(R_{\lambda}|v|\right)=0$ for $i=0,1$. Hence

$$
\int_{d_{0}}^{d_{1}}(\lambda+\varphi(x)) R_{\lambda}|v|(x) d x=\int_{d_{0}}^{d_{1}}|v(x)| d x,
$$

which shows that $\left\|\varphi R_{\lambda}|v|\right\|<\infty$ and $R_{\lambda} v \in L_{\varphi}^{1}$. Finally, from (4.8) it follows that

$$
A_{0}\left(R_{\lambda} v\right)=(\lambda+\varphi) R_{\lambda} v-v \in L^{1} .
$$

Now let $u \in \mathcal{D}(A)$ and $v:=\lambda u-A u$. Since $u \in L_{\varphi}^{1}$ and $A_{0} u \in L^{1}$, we have $v \in L^{1}$ and $R_{\lambda} v \in \mathcal{D}(A)$. Hence $w:=u-R_{\lambda} v \in \mathcal{D}(A)$ and $A w=\lambda w$. The general solution $w$ of this equation is of the form

$$
w(x)=c \frac{e^{-Q_{\lambda}(x)}}{g(x)}, \quad x \in E,
$$

where $c$ is a constant. Thus

$$
g(x)\left(u(x)-R_{\lambda} v(x)\right)=c e^{-Q_{\lambda}(x)} \quad \text { for } x \in E .
$$

If $\left|Q\left(d_{0}\right)\right|=+\infty$ and $c \neq 0$, then $c e^{-Q_{\lambda}}(\lambda+\varphi) / g$ is not integrable, which contradicts $u-R_{\lambda} v \in \mathcal{D}(A)$ and implies that $c=0$. If $Q_{\lambda}\left(d_{0}\right)=0$ then $l_{0}\left(u-R_{\lambda} v\right)=c$, which also gives $c=0$ and shows that $u=R_{\lambda} v$.

Proof of Theorem 5. Since $G\left(d_{1}\right)=+\infty$, the assumptions of Lemma 2 hold even when $\varphi=0$. Thus it is enough to show that $(A, \mathcal{D}(A))$ is the generator of $\{S(t)\}_{t \geq 0}$. Observe that $L^{1}=\operatorname{Im}(\lambda-A)$, by Lemma 2. Since (4.8) has a unique solution $u=R_{\lambda} v$, we have $R_{\lambda}=(\lambda-A)^{-1}$ for $\lambda>0$ and

$$
\|\lambda u-A u\|=\|v\| \geq \lambda\left\|R_{\lambda} v\right\|=\lambda\|u\| \quad \text { for } u \in \mathcal{D}(A) .
$$


The operator $\lambda R_{\lambda}$ is a positive contraction. By the Hille-Yosida theorem, the operator $A$ is a generator of a positive contraction semigroup. Let $\mathcal{A}$ be the generator of the semigroup $\{S(t)\}_{t \geq 0}$. It remains to prove that $\mathcal{A}=A$.

First, we will show that

$$
R(\lambda, \mathcal{A}) v=R_{\lambda} v \quad \text { for } v \in C_{c}(E), v \geq 0 .
$$

Let $v \in C_{c}(E), v \geq 0$. We have

$$
R(\lambda, \mathcal{A}) v=\lim _{t \rightarrow \infty} \int_{0}^{t} e^{-\lambda s} S(s) v d s
$$

where the integral is an element of $L^{1}$ such that

$$
\left(\int_{0}^{t} e^{-\lambda s} S(s) v d s\right)(x)=\int_{0}^{t} e^{-\lambda s} S(s) v(x) d s .
$$

Let $x \in E$ and $t>0$. Define $s_{*}(x)=\sup \left\{s>0: \pi_{-s} x \in E\right\}$ and note that $\pi_{-s_{*}(x)} x=d_{0}$. Making use of the formula for $S(t) v$ and the fact that $G\left(\pi_{-s} x\right)=G(x)-s$ when $\pi_{-s} x \in E$ leads to

$$
\int_{0}^{t} e^{-\lambda s} S(s) v(x) d s=\int_{0}^{t \wedge s_{*}(x)} e^{Q_{\lambda}\left(\pi_{-s} x\right)-Q_{\lambda}(x)} \frac{v\left(\pi_{-s} x\right) g\left(\pi_{-s} x\right)}{g(x)} d s .
$$

By a change of variables, we obtain

$$
\left|\int_{0}^{t} e^{-\lambda s} S(s) v(x) d s-R_{\lambda} v(x)\right| \leq \frac{1}{g(x)} e^{-Q_{\lambda}(x)} w(t, x),
$$

where

$$
w(t, x)= \begin{cases}\int_{d_{0}}^{\pi_{-t} x} e^{Q_{\lambda}(z)} v(z) d z & \text { if } t<s_{*}(x), \\ 0 & \text { if } t \geq s_{*}(x) .\end{cases}
$$

We have $w(t, x) \downarrow 0$ as $t \uparrow \infty$, thus

$$
\lim _{t \rightarrow \infty}\left\|\int_{0}^{t} e^{-\lambda s} S(s) v d s-R_{\lambda} v\right\|=0 .
$$

Since $C_{c}(E)$ is a dense subset of $L^{1}$ and both operators $\lambda R(\lambda, \mathcal{A})$ and $\lambda R_{\lambda}$ are positive contractions, they are identical. This shows that $\mathcal{D}(\mathcal{A})=\mathcal{D}(A)$ and $(\lambda-\mathcal{A}) u=(\lambda-A) u$ for $u \in \mathcal{D}(A)$, which completes the proof.

REMARK 1. Observe that if $G\left(d_{0}\right)=-\infty$ then the domain of $A_{0}$ is

$$
\mathcal{D}\left(A_{0}\right)=\left\{u \in L^{1}: g u \in A C, A_{0} u \in L^{1}\right\} .
$$


5. Asymptotic properties. In this section we assume that $g>0$ and $G\left(d_{1}\right)=+\infty$. Let $P: L^{1} \rightarrow L^{1}$ be a stochastic operator and let $\mathcal{C}$ be the operator

$$
\mathcal{C} u(x)=-\frac{d}{d x}(g(x) u(x))-\varphi(x) u(x)+P(\varphi u)(x), \quad x \in\left(d_{0}, d_{1}\right),
$$

defined on $\mathcal{D}(A)=\mathcal{D}\left(A_{0}\right) \cap L_{\varphi}^{1}$ with $\mathcal{D}\left(A_{0}\right)$ as in (4.5). By Section 3, it follows from Theorems 4 and 5 that there is a positive, strongly continuous, contraction semigroup $\{P(t)\}_{t \geq 0}$ on $L^{1}$ whose generator is an extension of the operator $(\mathcal{C}, \mathcal{D}(A))$. In this section we give sufficient conditions for $\{P(t)\}_{t \geq 0}$ to be a stochastic semigroup and study its asymptotic properties.

Define the operator $R_{0}$ on $\mathcal{D}\left(R_{0}\right)=\left\{v \in L^{1}: R_{0} v \in L^{1}\right\}$ by

$$
R_{0} v(x)=\int_{d_{0}}^{d_{1}} r_{0}(x, y) v(y) d y, \quad \text { where } \quad r_{0}(x, y)=\mathbf{1}_{\left(d_{0}, x\right)}(y) \frac{1}{g(x)} e^{Q(y)-Q(x)} .
$$

In general, $R_{0}$ may be an unbounded operator. We have

$$
\varphi(x) R_{0} v(x)=\int_{d_{0}}^{d_{1}} \varphi(x) r_{0}(x, y) v(y) d y
$$

for every $v \in \mathcal{D}\left(R_{0}\right)$. Since $Q$ is nondecreasing,

$$
\int_{d_{0}}^{d_{1}} \varphi(x) r_{0}(x, y) d x=\int_{y}^{d_{1}} \frac{\varphi(x)}{g(x)} e^{Q(y)-Q(x)} d x=1-e^{Q(y)-Q\left(d_{1}\right)} \leq 1
$$

for every $y \in E$. Thus the operator $\varphi R_{0}$ can be uniquely extended, with the same formula, to a positive contraction on $L^{1}$. Observe that $\varphi R_{0}$ is stochastic if and only if $Q\left(d_{1}\right)=+\infty$.

Define the operator $K$ by

$$
K u=P\left(\varphi R_{0}\right) u \text { for } u \in L^{1} .
$$

Since $P$ is a stochastic operator, $K$ is stochastic if and only if $Q\left(d_{1}\right)=+\infty$.

Theorem 6. Assume that $Q\left(d_{1}\right)=+\infty$.

If the operator $K$ has an invariant density $v_{*}>0$ a.e. then $\{P(t)\}_{t \geq 0}$ is a stochastic semigroup. Moreover, if $\{P(t)\}_{t \geq 0}$ is partially integral and $R_{0} v_{*} \in L^{1}$ then $\{P(t)\}_{t \geq 0}$ is asymptotically stable with invariant density $u_{*}=R_{0} v_{*} /\left\|R_{0} v_{*}\right\|$.

Conversely, if $\{P(t)\}_{t \geq 0}$ has an invariant density $u_{*} \in \mathcal{D}(A)$ then the density $P\left(\varphi u_{*}\right) /\left\|\varphi u_{*}\right\|$ is invariant for the operator $K$.

Proof. The operator $R_{1}=R(1, A)$ as defined in (4.7) satisfies $\left(\varphi R_{1}\right) u \leq$ $\left(\varphi R_{0}\right) u$ for every density $u$, which is due to the fact that $\varphi \geq 0$ and $r_{1} \leq r_{0}$. Since $P$ is a positive operator, we have $P(\varphi R(1, A)) v_{*} \leq v_{*}$ and $\{P(t)\}_{t \geq 0}$ is a stochastic semigroup, by Theorems 3 and 2. Now let $C$ be the generator 
of $\{P(t)\}_{t \geq 0}$. From (3.1) it follows that $R(1, C) u \geq R_{1} u$ for every density $u$. Since there is a $b(u) \in E$ such that $R_{1} u(x)>0$ for $x \geq b(u)$, the stochastic operator $R(1, C) u$ overlaps supports. Recall that $u_{*}$ is a stationary density for the semigroup $\{P(t)\}_{t \geq 0}$ if and only if $u_{*} \in \mathcal{D}(C)$ and $C u_{*}=0$. We have

$$
v_{*}=P\left(\varphi R_{0}\right) v_{*}=\left\|R_{0} v_{*}\right\| P\left(\varphi u_{*}\right) .
$$

It is easily seen that $u_{*} \in \mathcal{D}(A)$ and $A u_{*}=-v_{*} /\left\|R_{0} v_{*}\right\|$. Hence $C u_{*}=0$ and Theorem 1 applies.

Finally, suppose that $P(t) u_{*}=u_{*}$ for all $t \geq 0$ with $u_{*} \in \mathcal{D}(A)$. Since $\mathcal{D}(A) \subset L_{\varphi}^{1}$ and $C u_{*}=A u_{*}+P\left(\varphi u_{*}\right)$, we obtain $-A u_{*}=P\left(\varphi u_{*}\right)$. Thus $v:=P\left(\varphi u_{*}\right) \in L^{1}$ and $K v=P\left(\varphi R_{0} v\right)=-P\left(\varphi R_{0} A u_{*}\right)$. It is easily seen that $R_{0} A u_{*}=-u_{*}$ and we recover the claim.

REMARK 2. Observe that when $\varphi(x) \geq b>0$ for all $x$, then the operator $R_{0}$ is bounded, thus $R_{0}=R(0, A)=-A^{-1}$ and $R_{0} v_{*}$ is integrable. Moreover, if $\varphi$ is a constant function, $\varphi \equiv b$, then $(\mathcal{C}, \mathcal{D}(A))$ is the generator of $\{P(t)\}_{t \geq 0}$, by the Phillips perturbation theorem, and $K=P\left(b R\left(b, A_{0}\right)\right)$. In that case, the relation between the invariant densities for the operator $K$ and the semigroup $\{P(t)\}_{t>0}$ are a consequence of the equation $\mathcal{C} u_{*}=0$ which is now $A_{0} u_{*}-b u_{*}+b P u_{*}=0$ (see also [14]).

General sufficient conditions for existence of invariant densities for stochastic operators have been summarized by [12, Section 5] and [20]. We now discuss when a stochastic semigroup $\{P(t)\}_{t \geq 0}$ is partially integral. Let $P$ be the transition operator corresponding to a stochastic kernel $\mathcal{J}$. If there is a Borel measurable function $p: E \times E \rightarrow[0, \infty)$ such that

$$
\int_{E} \int_{E} p(x, y) \varphi(y) d y d x>0 \quad \text { and } \quad \mathcal{J}(x, B) \geq \int_{B} p(y, x) d y, \quad B \in \mathcal{B}(E),
$$

then $\{P(t)\}_{t \geq 0}$ is partially integral $[17,19]$. Now, if $P$ is the FrobeniusPerron operator corresponding to a nonsingular transformation $\sigma: E \rightarrow E$ then $\mathcal{J}(x, B)=\mathbf{1}_{B}(\sigma(x)), x \in E, B \in \mathcal{B}(E)$, and we have the following result.

Proposition 1 ([17, 19]). Let $\sigma: E \rightarrow E$ be continuously differentiable with $\sigma^{\prime}(x) \neq 0$ for almost every $x \in E$ and $P$ be the Frobenius-Perron operator corresponding to $\sigma$. Assume that the semigroup $\{P(t)\}_{t \geq 0}$ is stochastic. If there is $\bar{x} \in E$ such that $\varphi$ is continuous at $\bar{x}, \varphi(\bar{x})>0$, and $g(\sigma(\bar{x})) \neq \sigma^{\prime}(\bar{x}) g(\bar{x})$ then $\{P(t)\}_{t \geq 0}$ is partially integral.

6. Specific examples. As mentioned in the introduction, we were originally motivated by the work of Lasota et al. [13] who, in turn, were trying to understand the experimentally observed asymptotic properties of cell property densities in cellular populations in a growth phase. This led, naturally, 
to a consideration of dynamics such that $g>0$ for $x \in\left(d_{0}, d_{1}\right)$ as considered in Sections 4 and 5, and there are a number of concrete situations in this category to which we can apply our results. This section illustrates some of these. Several of the examples are drawn from the field of cell cycle kinetics, while others illustrate the necessity of certain assumptions.

Suppose first that the reset function is given by $\sigma(x)=x-\varrho(x)$, where $\varrho$ is a continuously differentiable function with $\varrho^{\prime}(x)<1$. Then the transition operator $P$ is of the form $P u(x)=\lambda^{\prime}(x) u(\lambda(x)) 1_{E}(\lambda(x))$, where $\lambda(x)=$ $\sigma^{-1}(x)$ is the inverse of $\sigma$, and we have the evolution equation

$$
\frac{\partial u(t, x)}{\partial t}=-\frac{\partial(g(x) u(t, x))}{\partial x}-\varphi(x) u(t, x)+u(t, \lambda(x)) \varphi(\lambda(x)) \lambda^{\prime}(x) 1_{E}(\lambda(x)) .
$$

If $Q\left(d_{1}\right)=\infty$ then the stochastic operator $K$ as defined in (5.1) is of the form

$$
K u(x)=\lambda^{\prime}(x) \frac{\varphi(\lambda(x))}{g(\lambda(x))} \mathbf{1}_{\left(d_{0}, d_{1}\right)}(\lambda(x)) \int_{d_{0}}^{\lambda(x)} e^{Q(y)-Q(\lambda(x))} u(y) d y
$$

which is the transition operator corresponding to (1.4), where $T(a, \tau)=$ $\sigma\left(Q^{-1}(Q(a)+\tau)\right)$.

EXAMPLE 1. Lasota and Mackey [11] considered a very general cell cycle model for the evolution of the distribution of "mitogen" at cell birth in which $g$ was a $C^{1}$ function on $[0,2 l)$ such that $g(x)>0$ for $x>0$ and $G(2 l)=\infty$, where $l$ is finite or not. Further, $\varphi$ was a continuous function on $[0,2 l)$ such that $\varphi(0)=0$,

$$
\liminf _{x \rightarrow 2 l} \varphi(x)>0 \quad \text { when } l<\infty \text { and } \liminf _{x \rightarrow \infty} q(x)>0,
$$

where $q(x)=\varphi(x) / g(x)$. In their model $\lambda(x)=2 x$. They were able to show that successive generations had densities evolving under the action of a stochastic operator

$$
K u(x)=2 q(2 x) \int_{0}^{2 x} \exp \left[-\int_{y}^{2 x} q(z) d z\right] u(y) d y \quad \text { for } 0<x<l
$$

and that $K$ is asymptotically stable.

EXAMPle 2. Building on this model Mackey et al. [16] took $g(x)=$ $x(2-x) / b$ and $\varphi(x)=S(x-1) \mathbf{1}_{(1,2)}(x)$ with $b, S>0$ and $x \in(0,2)$ to fit a number of in vitro cell cycle data sets. With these choices for $g$ and $\varphi$ it is straightforward to show that the unique stationary density of mitogen is

$$
v_{*}(x)=S b \cdot 2^{S b}(x-1 / 2)[x(1-x)]^{S b / 2-1} \text { for } x \in(1 / 2,1) .
$$


Assume that $E=\left(d_{0}, \infty\right)$ and $Q(\infty)=\infty$. We can rewrite the operator $K$ given by (6.1) in the form

$$
K u(x)=\int_{d_{0}}^{\lambda(x)}-\frac{\partial}{\partial x}\left(e^{Q(y)-Q(\lambda(x))}\right) u(y) d y .
$$

Asymptotic properties of this operator have been well studied [8, 13, 3, 18].

Proposition $2([8])$. Assume that $\varphi(x)>0$ for $x>d_{0}$ and $Q\left(d_{0}\right)=0$. If

$$
\liminf _{x \rightarrow \infty}(Q(\lambda(x))-Q(x))>1
$$

then $K$ as defined in (6.2) has a strictly positive invariant density and if $Q(\lambda(x))-Q(x) \leq 1$ for all $x>d_{0}$ it has no invariant density.

Remark 3. The assumption $Q\left(d_{0}\right)=0$ cannot be omitted in Proposition 2 , as the following example shows.

Let $E=(0, \infty), \lambda(x)=2 x$, and $Q(x)=b \log x$. The operator $K$ is now

$$
K u(x)=\frac{b}{2^{b} x^{b+1}} \int_{0}^{2 x} y^{b} u(y) d y .
$$

If we take $u(x)=1 / x$ then $K u(x)=u(x)$ for all $b>0$. This shows that $K$ has a subinvariant function which is strictly positive and not integrable. Since $K$ overlaps supports, it has no invariant density [18, Remark 6] for any $b>0$, but condition (6.3) holds whenever $b \log 2>1$.

EXAMPLE 3. Consider the following functions:

$$
g(x)=k, \quad k>0, \quad \varphi(x)=p x^{\alpha}, \quad p>0, \alpha>-1, \quad \lambda(x)=2 x, \quad x>0 .
$$

We have $G(x)=k x$ and $Q(x)=b x^{\alpha+1} /(\alpha+1)$, where $b=p / k$. The operator $K$ has a strictly positive invariant density, by Proposition 2. Thus the semigroup $\{P(t)\}_{t \geq 0}$ is stochastic, by Theorem 6 , and it is partially integral, by Proposition 1 , since $g(x / 2) \neq g(x) / 2$ for all $x$. The domain of the operator $A$ is

$$
\mathcal{D}(A)=\left\{u \in L^{1}[0, \infty): u(0)=0, u \in A C, u^{\prime} \in L^{1}, \int_{0}^{\infty} x^{\alpha}|u(x)| d x<\infty\right\} .
$$

The stationary density $u_{*} \in \mathcal{D}(A)$ for the semigroup $\{P(t)\}_{t \geq 0}$ is a solution of the equation

$$
u^{\prime}(x)=-b x^{\alpha} u(x)+2 b(2 x)^{\alpha} u(2 x), \quad x>0,
$$

and is given by

$$
u_{*}(x)=\sum_{n=0}^{\infty} c_{n} e^{-Q\left(2^{n} x\right)}, \quad \text { where } \quad c_{n}=\frac{2^{\alpha+1}}{1-2^{n(\alpha+1)}} c_{n-1}, \quad n \geq 1,
$$


and $c_{0}$ is a normalizing constant. The stationary density $v_{*}$ for the operator $K$ is given by $v_{*}(x)=c x^{\alpha} u_{*}(2 x)$, where $c$ is a normalizing constant.

We continue with the above example, but now we take $\alpha=-1$ and show that for certain values of the parameter $b$ the semigroup is stochastic and for others it is not. Observe that we have $Q(x)=b \log x$, thus the operator $K$ is the operator from Remark 3 and it is not asymptotically stable.

EXAmple 4. Let the functions $g$ and $\lambda$ be as in Example 3. Let $Q(x)=$ $b \log x$, where $b=p / k$. We have

$$
P\left(\varphi R_{1}\right) u(x)=\frac{b e^{-2 k x}}{2^{b} x^{b+1}} \int_{0}^{2 x} e^{k y} y^{b} u(y) d y .
$$

If we take $u(x)=x^{\beta-1} e^{-k x}$ then $u \in L^{1}$ for $\beta>0$ and

$$
P\left(\varphi R_{1}\right) u(x)=\frac{b 2^{\beta}}{b+\beta} u(x) e^{-k x} .
$$

Assume that $b \log 2<1$. Since we can find $\beta>0$ such that $b 2^{\beta} \leq b+\beta$, the operator $P\left(\varphi R_{1}\right)$ has a subinvariant strictly positive density, which shows that the semigroup $\{P(t)\}_{t \geq 0}$ is stochastic, by Theorem 3 .

Assume now that $b \log 2>1$ and take $k=1$. If we go back to (1.4) then

$$
a_{n}=\frac{1}{2} a_{n-1} e^{\tau_{n} / b} \quad \text { and } \quad \mathbb{E}\left(a_{n}^{\gamma}\right)=\frac{\mathbb{E}\left(a_{0}^{\gamma}\right)}{2^{n \gamma}}\left(\frac{b}{b-\gamma}\right)^{n} .
$$

We can find $\gamma<1$ such that $b 2^{-\gamma}<b-\gamma$. Since $t_{n}=a_{n}-a_{0}+\sum_{i=1}^{n} a_{i}$, this shows that $\sup _{n} \mathbb{E}\left(t_{n}^{\gamma}\right)<\infty$, so that the process is defined only up to a finite random time and $\{P(t)\}_{t \geq 0}$ cannot be stochastic.

Example 5. Tyson and Hannsgen [24] in their cell cycle model consider a special case of the model of [11] in which they let $E=(\sigma, \infty)$, where $\sigma<1$, and consider the following functions:

$$
g(x)=k x, \quad \varphi(x)=\left\{\begin{array}{ll}
0, & x<1, \\
p, & x \geq 1,
\end{array} \quad \sigma(x)=\sigma x .\right.
$$

They show that the unique steady state $v_{*}$ is given by

$$
v_{*}(x)=\frac{r-1}{\sigma}\left(\frac{x}{\sigma}\right)^{-r},
$$

where the exponent $r>1$ must satisfy

$$
b-(r-1)=b \sigma^{r-1} \quad \text { and } \quad b \ln \frac{1}{\sigma}>1, \quad \text { where } b=\frac{p}{k} .
$$

In this example we have $g(\sigma x)=\sigma g(x)$ for all $x$ and the semigroup $\{P(t)\}_{t \geq 0}$ is not partially integral. Although it has a unique strictly positive stationary density, it is not asymptotically stable due to a possible synchronization [5]. 
We conclude this section with an example when the reset function $\sigma$ depends additionally on an external variable. Let $E=(0, \infty), \Theta=(0,1), \nu$ be a measure on $\Theta$ with a density $h$, and the reset function $\sigma$ be of the form $\sigma(x, \theta)=x-\theta x$. Then the transition operator $P$ has the kernel

$$
p(x, y)=1_{(0, x)}(y) \psi\left(\frac{x}{y}\right) \frac{1}{y}, \quad \text { where } \psi(\theta)=h(1-\theta),
$$

and the evolution equation is

$$
\frac{\partial u(t, x)}{\partial t}=-\frac{\partial g(x) u(t, x)}{\partial x}-\varphi(x) u(t, x)+\int_{x}^{\infty} \psi\left(\frac{x}{y}\right) \frac{\varphi(y)}{y} u(t, y) d y .
$$

The operator $K$ has the kernel

$$
k(x, y)=\int_{\max \{x, y\}}^{\infty} \psi\left(\frac{x}{z}\right) \frac{\varphi(z)}{z g(z)} e^{Q(y)-Q(z)} d z, \quad x, y \in(0, \infty) .
$$

EXAmple 6. Suppose that $\varphi(x) / g(x)=b x^{\alpha}$ for all $x>0$, where $b>0$ and $\alpha>-1$. We have $Q(x)=b x^{\alpha+1} /(\alpha+1)$. We provide the form of the invariant density for $K$ when $\psi(z)=\beta z^{\beta-1}$ for $z \in[0,1]$ and $\beta>0$. It is easily seen that the invariant density is of the form

$$
v_{*}(x)=\frac{b^{\gamma}}{(\alpha+1)^{1+\gamma} \Gamma(\gamma)} x^{\beta-1} e^{-Q(x)}, \quad \gamma=\frac{\beta}{\alpha+1} .
$$

We have

$$
R_{0} v_{*}(x)=\frac{x v_{*}(x)}{\beta g(x)} .
$$

If $R_{0} v_{*} \in L^{1}$ then $\{P(t)\}$ is an asymptotically stable stochastic semigroup by Theorem 6 . For example when $\alpha=0$ then $v_{*}$ is the gamma distribution, while if $\alpha=\beta=1$ then

$$
v_{*}(x)=\frac{\sqrt{b}}{2 \sqrt{2 \pi}} e^{-b x^{2} / 2} .
$$

7. Decay instead of growth. We assumed in Sections 4 and 5 that $g(x)>0$ for $x \in\left(d_{0}, d_{1}\right)$, and illustrated the applicability of our results to concrete situations in Section 6. In this section we discuss a situation when instead of growth there is degradation, so now we suppose that $g(x)<0$ for $x \in\left(d_{0}, d_{1}\right)$. Results analogous to those of Sections 4 and 5 with similar proofs are valid in this case, and we illustrate the applicability of these to models for the stochastic regulation of gene expression.

Let the functions $G, Q$ be defined as in (4.1). Observe that now $G$ is decreasing and $Q$ is nonincreasing. If $G\left(d_{0}\right)=+\infty$ then $\pi_{t}(E) \subseteq E$ for all $t \geq 0$ and $t \mapsto \pi_{t} x$ is decreasing. Let $\left\{P_{0}(t)\right\}_{t \geq 0}$ and $\{S(t)\}_{t \geq 0}$ be as in (4.2) and (4.3). Thus, if $g<0$ and $G\left(d_{0}\right)=+\infty$ the conclusions of Theorem 4 
remain valid. The analogue of Theorem 5 with the same method of proof reads as follows.

TheOREM 7. If $g<0$ and $G\left(d_{0}\right)=+\infty$ then the operator $A_{0}$ defined on the domain

$$
\mathcal{D}\left(A_{0}\right)=\left\{u \in L^{1}: g u \in A C, A_{0} u \in L^{1}, \lim _{x \uparrow d_{1}} g(x) u(x)=0\right\}
$$

is the generator of the semigroup $\left\{P_{0}(t)\right\}_{t>0}$ and the operator $A$ defined on $\mathcal{D}(A)=\mathcal{D}\left(A_{0}\right) \cap L_{\varphi}^{1}$ is the generator of $\{S(t)\}_{t \geq 0}$.

Moreover, the resolvent of the operator $A$ is of the form

$$
R(\lambda, A) v(x)=\int_{x}^{d_{1}} \frac{1}{|g(x)|} e^{Q_{\lambda}(y)-Q_{\lambda}(x)} v(y) d y, \quad v \in L^{1} .
$$

The operator $R_{0}$ is now defined with the help of the function

$$
r_{0}(x, y)=\mathbf{1}_{\left(x, d_{1}\right)}(y) \frac{1}{|g(x)|} e^{Q(y)-Q(x)}
$$

and the assertions of Theorem 6 remain valid under the assumption that $g<0$ and $G\left(d_{0}\right)=Q\left(d_{0}\right)=+\infty$.

In particular, if the operator $P$ has kernel $p$ then the operator $K$ has the kernel

$$
k(x, y)=\int_{d_{0}}^{y} p(x, z) \frac{\varphi(z)}{|g(z)|} e^{Q(y)-Q(z)} d z
$$

and is stochastic if and only if $Q\left(d_{0}\right)=\infty$.

Let $E=\Theta=(0, \infty)$ and $\sigma(x, \theta)=x+\theta$. Then the operator $P$ is the convolution operator with the measure $\nu$, i.e. if $\zeta_{1}$ has density $u$ and $\theta_{1}$ has distribution $\nu$ then $P u$ is the density of $\zeta_{1}+\eta_{1}$. Assume that $\nu$ has a density $h$. Then

$$
p(x, y)=\mathbf{1}_{(0, x)}(y) h(x-y),
$$

so that our evolution equation is

$$
\frac{\partial u(t, x)}{\partial t}=-\frac{\partial g(x) u(t, x)}{\partial x}-\varphi(x) u(t, x)+\int_{0}^{x} h(x-y) \varphi(y) u(t, y) d y .
$$

EXAMPLE 7. Friedman et al. [6] have considered stochastic aspects of gene expression following from bursts of protein production, and their formulations are special cases of our results. Identifying their $w$ and $c$ with $w(x-y) d y=h(x-y) d y-\delta_{x}(d y)$ and $\varphi(x)=k_{1} c(x)$ and taking $g(x)=-\gamma x$, our equation becomes identical, in a steady state situation to their equation (6),

$$
-\frac{\partial \gamma x u_{*}(x)}{\partial x}=\int_{0}^{x} \varphi(y) u_{*}(y) w(x-y) d y
$$


Then, following [6], let $h(y)=(1 / b) e^{-y / b}$ be the exponential distribution and let, in their first model, $\varphi(x)=k_{1}$. Then the equation, as they have shown, has as a solution the density of the gamma distribution. In considering the second model of [6] that treated transcription factor regulation of its own transcription, we are led to consider the function

$$
\varphi(x)=k_{1} \frac{1}{1+x^{\alpha}}+k_{1} \varepsilon .
$$

As they have shown, the corresponding density is given by

$$
u_{*}(x)=c x^{a(1+\varepsilon)-1} e^{-x / b}\left[\frac{1}{1+x^{\alpha}}\right]^{a / \alpha},
$$

where $c$ is a normalizing factor.

Acknowledgments. This work was supported by the Natural Sciences and Engineering Research Council (NSERC, Canada), by the Mathematics of Information Technology and Complex Systems (MITACS, Canada), and by the State Committee for Scientific Research (Poland) Grant N N201 0211 33 (MT-K). This research was partially carried out while MCM was visiting the Institut für theoretische Physik, Universität Bremen, and while MT-K was visiting McGill University.

\section{References}

[1] J. Banasiak, On an extension of the Kato-Voigt perturbation theorem for substochastic semigroups and its application, Taiwanese J. Math. 5 (2001), 169-191.

[2] J. Banasiak and L. Arlotti, Perturbations of Positive Semigroups with Applications, Springer Monogr. Math., Springer, London, 2006.

[3] K. Baron and A. Lasota, Asymptotic properties of Markov operators defined by Volterra type integrals, Ann. Polon. Math. 58 (1993), 161-175.

[4] A. Bobrowski, T. Lipniacki, K. Pichór, and R. Rudnicki, Asymptotic behavior of distributions of $m R N A$ and protein levels in a model of stochastic gene expression, J. Math. Anal. Appl. 333 (2007), 753-769.

[5] O. Diekmann, H. J. A. M. Heijmans, and H. R. Thieme, On the stability of the cell size distribution, J. Math. Biol. 19 (1984), 227-248.

[6] N. Friedman, L. Cai, and X. Xie, Linking stochastic dynamics to population distribution: An analytical framework of gene expression, Phys. Rev. Lett. 97 (2006), $168302-1 / 4$.

[7] G. Frosali, C. V. M. van der Mee, and F. Mugelli, A characterization theorem for the evolution semigroup generated by the sum of two unbounded operators, Math. Methods Appl. Sci. 27 (2004), 669-685.

[8] H. Gacki and A. Lasota, Markov operators defined by Volterra type integrals with advanced argument, Ann. Polon. Math. 51 (1990), 155-166.

[9] D. Gillespie, Exact stochastic simulation of coupled chemical reactions, J. Phys. Chem. 81 (1977), 2340-2361. 
[10] T. Kato, On the semi-groups generated by Kolmogoroff's differential equations, J. Math. Soc. Japan 6 (1954), 1-15.

[11] A. Lasota and M. C. Mackey, Globally asymptotic properties of proliferating cell populations, J. Math. Biol. 19 (1984), 43-62.

[12] —, -, Chaos, Fractals, and Noise, Appl. Math. Sci. 97, Springer, New York, 1994.

[13] A. Lasota, M. C. Mackey, and J. Tyrcha, The statistical dynamics of recurrent biological events, J. Math. Biol. 30 (1992), 775-800.

[14] A. Lasota and J. Traple, Invariant measures related with Poisson driven stochastic differential equation, Stochastic Process. Appl. 106 (2003), 81-93.

[15] T. Lipniacki, P. Paszek, A. Marciniak-Czochra, A. R. Brasier, and M. Kimmel, Transcriptional stochasticity in gene expression, J. Theoret. Biol. 238 (2006), 348367.

[16] M. C. Mackey, M. Santavy, and P. Selepova, A mitotic oscillator with a strange attractor and distributions of cell cycle times, in: Nonlinear Oscillations in Biology and Chemistry (Salt Lake City, UT, 1985), Lecture Notes in Biomath. 66, Springer, Berlin, 1986, 34-45.

[17] K. Pichór and R. Rudnicki, Continuous Markov semigroups and stability of transport equations, J. Math. Anal. Appl. 249 (2000), 668-685.

[18] R. Rudnicki, On asymptotic stability and sweeping for Markov operators, Bull. Polish Acad. Sci. Math. 43 (1995), 245-262.

[19] R. Rudnicki and K. Pichór, Markov semigroups and stability of the cell maturity distribution, J. Biol. Systems 8 (2000), 69-94.

[20] R. Rudnicki, K. Pichór, and M. Tyran-Kamińska, Markov semigroups and their applications, in: Dynamics of Dissipation, P. Garbaczewski and R. Olkiewicz (eds.), Lecture Notes in Phys. 597, Springer, Berlin, 2002, 215-238.

[21] J. Traple, Markov semigroups generated by Poisson driven differential equations, Bull. Polish Acad. Sci. Math. 44 (1996), 161-182.

[22] M. Tyran-Kamińska, Substochastic semigroups and densities of piecewise deterministic Markov processes, http://arxiv.org/abs/0804.4889, preprint, 2008.

[23] J. Tyrcha, Asymptotic stability in a generalized probabilistic/deterministic model of the cell cycle, J. Math. Biol. 26 (1988), 465-475.

[24] J. J. Tyson and K. B. Hannsgen, Cell growth and division: a deterministic/probabilistic model of the cell cycle, ibid. 23 (1986), 231-246.

[25] J. Voigt, On substochastic $C_{0}$-semigroups and their generators, Transport Theory Statist. Phys. 16 (1987), 453-466.

Departments of Physiology, Physics \& Mathematics and Centre for Nonlinear Dynamics

McGill University

3655 Promenade Sir William Osler

Montreal, QC, Canada, H3G 1Y6

E-mail: mackey@cnd.mcgill.ca
Institute of Mathematics Polish Academy of Sciences Bankowa 14 40-007 Katowice, Poland and

Institute of Mathematics University of Silesia Bankowa 14 40-007 Katowice, Poland E-mail: mtyran@us.edu.pl 